\title{
Argumentative Topoi als Ressource für die Argumentationsschulung im DaF-Unterricht
}

\author{
Abdel-Hafiez Massud
}

\begin{abstract}
Zusammenfassung
Dieser Beitrag beschäftigt sich mit der Argumentationskompetenz im DaF-Unterricht und konzentriert sich dabei auf die argumentativen Topoi im Sinne der Argumentationsschemata, die auf Aristoteles zurückgehen. Nach der theoretischen Etablierung der Begriffsdefinition und der Einführung in die vorhandenen Typologien dieser Argumentationsmuster wird ein mehrschrittiger didaktischer Ansatz vorgestellt, wie die argumentativen Topoi in die DaF-Didaktik mündlich und schriftlich ertragreich aufgenommen werden können. Der Ansatz wird nicht nur theoretisch vorgestellt, sondern auch an einem praktischen Beispiel erläutert, nämlich an dem Gedicht »Was gesagt werden muss« von Günter Grass und an einigen diesbezüglichen Online-Kommentaren deutscher Muttersprachler. Es wird aufgezeigt, dass die Didaktisierung dieser Topoi in einen argumentationsorientierten einheitlichen DaF-Unterricht mit Erfolg integriert werden kann und als Zugang zur vertiefenden Landeskunde und als Stimulator zum bewussten strategischen Spracherwerb dienen kann.
\end{abstract}

\section{Vorbemerkung}

Argumentation, ob schriftlich oder mündlich, ist stets eine partnerbezogene Sprachhandlung, bei der Sprecher versuchen, einander dazu zu bringen, etwas zu tun bzw. zu akzeptieren oder etwas zu unterlassen bzw. Abstand von etwas zu nehmen. Argumentation ist daher ein kommunikativer Prozess, der unter dem Einsatz der Ganzheit des Sprechers auf die Ganzheit des Adressaten abzielt. Dieser Andere ist erst mit der kommunikativen Wende im DaF-Unterricht in den siebziger Jahren des letzten Jahrhunderts in den Fokus des Interesses gerückt. Denn der kommunikative Ansatz zielt ja darauf ab, den DaF-Lerner in die Lage zu ver- 
setzen, die Fremdsprache situationsangemessen und zielgerichtet (Huneke/Steinig 2002: 170 f.) anzuwenden. Savignon (1983) hob hervor, dass zu dieser kommunikativen Kompetenz nicht nur die Fertigkeiten (Hörverstehen, Sprechen, Leseverstehen und Schreiben) gehören, sondern auch die vier Komponenten der grammatischen, soziolinguistischen, diskursiven und strategischen Kompetenz. Die Diskurskompetenz bezieht sich nach manchen Autoren jedoch nur auf die

»Fähigkeit des Individuums, unterschiedliche Texte zu verstehen und zu produzieren. Der Lerner muss beim Sprechen und beim Hören in der Lage sein, grammatikalische Formen und Bedeutungen miteinander im Diskurs zu verbinden und zu verstehen und nicht als einzelne Äußerungen aufzufassen.«(Al-Nasser 2011: $27 \mathrm{f}$.).

Die strategische Kompetenz betont die Notwendigkeit, dass der Lerner ${ }^{1}$ die »verbalen und nonverbalen Kommunikationsstrategien beherrscht, um bei Schwierigkeiten kommunikative Situationen zu bewältigen « (ebd.: 28). Eine ausgesprochen eingehende Behandlung der argumentativen Kompetenz fehlt jedoch in diesem kommunikativen Ansatz des DaF-Unterrichts. Erst mit dem Gemeinsamen Europäischen Referenzrahmen für Sprachen begann man, die Argumentationskompetenz als eine besondere Teil-Kompetenz der fortgeschrittenen kommunikativen Kompetenz sensibilisierter wahrzunehmen. Im Wesentlichen sieht der Referenzrahmen die Entwicklung der argumentativen Kompetenz ab dem Niveau C1 vor, wo der Deutsch-Lerner mindestens 1000 Unterrichtsstunden in der deutschen Sprache absolviert hat und wo von ihm zu erwarten ist, dass er bereits über ausreichende Sprachkompetenz verfügt. So lesen wir im Referenzrahmen unter dem Niveau C1 zur Beurteilung der mündlichen Kommunikation:

»Ich kann mich schriftlich klar und gut strukturiert ausdrücken und meine Ansicht ausführlich darstellen. Ich kann in Briefen, Aufsätzen oder Berichten über komplexe Sachverhalte schreiben und die für mich wesentlichen Aspekte hervorheben. Ich kann in meinen schriftlichen Texten den Stil wählen, der für die jeweiligen Leser angemessen ist." (Europarat 2001: 36)

Dennoch kommt der Entwicklung der argumentativen Kompetenz der DaF-Lerner im Gemeinsamen Europäischen Referenzrahmen nicht die ihr gebührende hohe Stellung zu, die gleichermaßen für Lehrkräfte und Lehrwerke die erforderlichen markanten Handlungskonsequenzen nach sich ziehen würde; die Lehrwerke letzten Standes, welche auf der Basis der Kriterien des Gemeinsamen Europäischen Referenzrahmens entwickelt worden sind, legen hiervon ein sprechendes Zeugnis ab. Zwar kommen in Lehrwerken wie etwa dem am meisten gelobten Tangram und Tangram aktuell bestimmte argumentative Sprachhandlun-

${ }^{1}$ Der Einfachheit halber beschränkt sich dieser Beitrag auf die Verwendung des generischen Maskulinums. Es sind damit aber immer Frauen und Männer gleichermaßen gemeint. 
gen und Begründungsstrukturen vor (vgl. Al-Nasser 2011). Diese sind aber durch die Vermittlung von Sprachmitteln bedingt zu verstreut, zu inkohärent und zu konzeptionslos, als dass eine bewusste systematische Schulung der Argumentation für die DaF-Lerner daraus erwachsen könnte. Insofern hinkt der DaF-Unterricht hinter den tatsächlichen Erwartungen des Fremdsprachenlernens in einer globalisierten und multikulturellen mündigen Welt, hinter der linguistischen kontrastiven Argumentationsforschung und auch hinter dem Stand der Argumentationsschulung im muttersprachlichen Deutschunterricht her, wo diese Schulung und die Erziehung zum kritischen Denken schon ab der 6. Klasse aufwärts seit den 70er Jahren des vergangenen Jahrhunderts bewusst und systematisch betrieben wird (vgl. z. B. Kopperschmidt 1975, Vinçon 1990, Ockel 1992, Winkler 2003). Dabei wäre die Schulung zur Argumentation im DaF-Unterricht kein Hindernis auf dem Weg zum schnellen und effektiven Spracherwerb und der Entwicklung der Sprachkompetenz, sondern eher ein großer Stimulator (vgl. Connor 1987; Massie 2005), da sich ein Deutschlerner in der konkreten Situation der Argumentation gezwungen sieht, sämtliche Sprachregister sowie sein gesamtes Kulturund Weltwissen einzusetzen, und die Sprache und ihren Nutzen in authentischen oder quasi-authentischen Argumentationssituationen erfährt, wie Ohlinger (2003) das in ihrer umfangreichen Studie über die Argumentationsfähigkeiten und Argumentationsdefizite litauischer Deutsch-Lerner und Keller (2013: insbesondere 93-97) in seiner Didaktisierung argumentativer Schreibkompetenz fürs Englische bestätigen konnten. Somit ist es verständlich, dass die tatsächliche Argumentationsschulung im DaF-Unterricht nur auf einem fortgeschrittenen Niveau ab C1 oder einem äquivalenten Niveau erfolgen kann, wo die sprachlichen Grundvoraussetzungen für die Teilnahme an einer Argumentation in der Zielsprache Deutsch erfüllt sein sollten. Zu diesen Voraussetzungen, die sowohl für die schriftliche als auch für die mündliche Argumentation gelten, gehören zunächst sämtliche kommunikative Fertigkeiten sowie die von Savignon oben angesprochenen Einzelkompetenzen. Bei der mündlichen Argumentation erübrigt es sich, darauf hinzuweisen, dass eine elementare Voraussetzung für eine gelungene und effektive Argumentation neben der Beherrschung der Techniken der Gesprächsorganisation und der nonverbalen Techniken auch die ausgebildete fließende Aussprache (Prosodie) des Deutschen ist, welche in den DaF-Lehrwerken immer noch stiefmütterlich (vgl. Al-Nasser 2011) behandelt wird. Sowohl in der schriftlichen als auch in der mündlichen Argumentation wird der Erfolg des Argumentierens zudem wesentlich davon abhängen, ob die argumentierende Person über einen ausreichenden und variablen Wortschatz, über eine zielorientierte Wortwahl, über eine ausgeprägte grammatische Kompetenz und über die Fähigkeit verfügt, kultursensible Argumente zu formulieren, da Argumentation gegenüber einer fremden Kultur ja nicht nur eine kommunikative Handlung ist, sondern auch eine (inter-)kulturelle Leistung darstellt (vgl. Philipsen 1992). 
Im vorliegenden Beitrag geht es darum, die Fertigkeit der DaF-Lerner im Umgang mit den argumentativen Topoi im Sinne von Argumentationsmustern auszubilden und diese Fertigkeit im kommunikativ orientierten DaF-Unterricht explizit unter Beweis zu stellen, denn die Argumentationsmuster stellen neben der sprachlichen Kompetenz ein Kernelement der Alltagsargumentation dar.

\section{Argumentative Topoi und ihre Relevanz für den DaF-Unterricht}

Die folgenden Ausführungen sollen nur insoweit einen Überblick über den Begriff argumentativer Topos und dessen Relevanz für die Alltagsargumentation bieten, als er für die Didaktisierung der Argumentation im DaF-Unterricht verwertbar zu machen ist und dem empirischen DaF-Unterricht als verständliche theoretische Handhabe dient. Mit den folgenden Ausführungen ist weder eine philosophische noch eine rhetorische Abhandlung intendiert.

Aristoteles hat den Begriff Topos zwar nicht verbindlich (Drehe 2011: 129) definiert, aber Verwendungsbeispiele hinterlassen, mit denen sich viele Autoren (vgl. z. B. Bornscheuer 1976; Kienpointner 1992; Slomkowski 1997; Wengeler 2003; Rubinelli 2009) auseinandergesetzt haben. Allenthalben wird bestätigt, dass der Begriff unterschiedliche Konzepte bezeichnet, die miteinander nicht viel zu tun haben (Hoppmann: 2009: 637). Um so mehr ist es für den vorliegenden Beitrag von zentraler Bedeutung, zu konstatieren, was hier unter argumentativem Topos verstanden wird, nämlich ein Argumentationsschema für einen Argumentationsschritt (vgl. Hoppmann 2008; Wengeler 1995). Der Topos ermöglicht den logischen Übergang von einem nicht umstrittenen Sachverhalt zu einer Konklusion, damit die in dieser Konklusion enthaltene Behauptung Gültigkeit und Akzeptanz erlangt. Das soll folgendes Beispiel konkreter verdeutlichen:

\section{Bildung ist notwendig (unumstrittene Aussage bzw. Prämisse) \\ Sprachausbildung ist Bildung (Topos)}

\section{Sprachausbildung ist notwendig (Konklusion)}

In Anlehnung an Aristoteles (s. Wagner/Rapp 2004) spricht Hoppmann (2009: 637) von einer doppelten Funktion der argumentativen Topoi:

»Zum einen helfen sie zu einer angestrebten Konklusion die geeigneten Prämissen aufzufinden (heuristische Funktion) und zum anderen bilden sie das Argumentationsschema, mit dessen Hilfe die Zustimmung von einer Prämisse auf die Konklusion übertragen werden kann (probative Funktion).«

Die einfache rhetorisch-topische Argumentation hat somit folgende Struktur: ${ }^{1}$

1 Vgl. ähnliche Darstellungsformen auch bei Ueding/Steinbrink (1994: 235). 


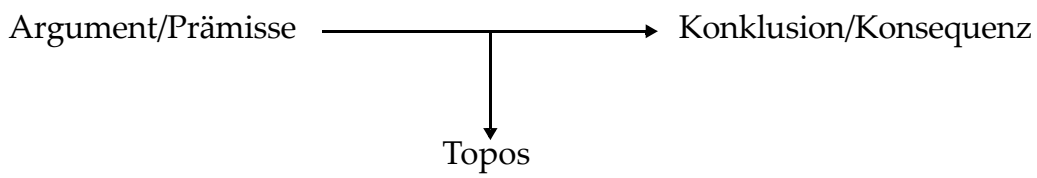

Natürlich sprechen wir in der Alltagssprache nicht in solchen Formeln; hier sehen wir nur die Explizierung, die wir zu den Zwecken dieses Beitrags vornehmen und die man in der Alltagssprache bei Missverständnissen ebenfalls vornehmen kann. Diese Explizierung in drei Schritten wäre als ein »formallogischer Syllogismus « 1 zu bezeichnen. Ohne diese Explizierung hat man es mit einem »Enthymem « ${ }^{2} \mathrm{zu}$ tun. Ein Enthymem ist in heutiger ${ }^{3}$ Verwendung somit ein Syllogismus in einem konkreten Kontext ohne formallogische Explizierung. Somit stellen Syllogismen und Enthymeme heute keine grundsätzlichen Gegensätze, sondern verwendungsbedingte gleiche Phänomene dar. In diesem Sinne schreibt Christof Rapp (2000) in seinem Kommentar zur Aristoteles-Rhetorik:

»damit wird bestätigt, dass ein Enthymem im selben Sinn ein syllogismus ist wie derjenige syllogismus, für den der Dialektiker zuständig ist. [...] Somit wird für das Enthymem im Grunde keine andere Differenz benannt als diejenige, dass das Enthymem ein syllogismus im rhetorischen Gebrauch ist. « ${ }^{4}$

In der Regel sprechen die Menschen im Alltag enthymematisch, d.h. ohne den Topos explizit zu nennen, wie etwa im folgenden Beispiel, wo die Information, dass Sprachausbildung Teil der allgemeinen Bildung ist, »in Gedanken « vom Rezipienten selbst erbracht wird:

Sprachausbildung ist notwendig, denn Bildung ist notwendig.

Enthymeme werden somit im Rahmen einer »kooperativen Interaktion « zwischen dem Sprechenden und dem Rezipienten konstruiert und gewinnen um so mehr an Überzeugungskraft. Der Topos entspricht daher »der Schlussregel« im viel zitierten Argumentationsmodell Stephen Toulmins (1958/1975) ${ }^{5}$ und schöpft seine

1 Graff (2008: 718 f.); mehr zur begrifflichen Unterscheidung von Enthymem, Syllogismus und Topos finden sich auch in Rapp (2000: 15-35).

2 Ueding/Steinbrink (2011: $267 \mathrm{f}$.).

3 Ueding/Steinbrink (2011: 267) machen darauf aufmerksam, dass das Enthymem in der antiken Rhetorik sehr viel mehr als einen »verkürzten Syllogismus « bedeutet, »nämlich eine besondere Art des Schlussverfahrens, das nicht formallogisch zu sein brauchte, sondern als Teil der Topik verstanden wurde «.

4 Flashar (2002: 228-229).

5 Toulmins Modell besteht aus 6 Kategorien: Der These/Konklusion (C), dem Argument (A), der Schlussregel ( $\mathrm{W}=$ Warrant), der Stützung ( $\mathrm{B}$ = backing), dem Modaloperator ( $\mathrm{Q}$ $=$ Qualifier) und schließlich der Ausnahmebedingung $(\mathrm{R}=$ rebuttal). In konkreten argumentativen Texten werden in der Regel aber die These und die Argumente am häufigs- 
Überzeugungskraft nicht etwa von seiner zwingenden Richtigkeit, sondern von seiner alltäglich anerkannten Plausibilität (Wengeler 1995: 38). Gerade dieses Argumentationsschema von Toulmin, dem der Topos / die Schlussregel zugrunde liegt, ist für Brinker (1997: 74-76) eine Wesensvoraussetzung für die Klassifizierung der Texte als »argumentative Texte « schlechthin. $\mathrm{Zu}$ den weiteren Elementen, welche für die Argumentationshaftigkeit von Texten sprechen, zählt Kienpointner (1992: 237-239) explizite Äußerungen, z. B. »Du verwechselst Ursache und Wirkung «, bestimmte Partikeln und Konnektoren sowie den verbalen und situativen Kontext des jeweiligen Textes. Diese sprachlichen und kontextuellen Elemente weisen zugleich auf das Vorliegen von Argumentationsmustern im Sinne der Topoi hin, zumal der Topos als Schlussregel wie oben dargestellt normalerweise nicht explizit genannt wird, sondern implizit bleibt, was wiederum eine hohe Sprachkompetenz voraussetzt.

Hilfreich und erhellend sowohl für die kontrastive Linguistik der Argumentation als auch für den DaF-Unterricht und die interkulturelle Argumentation ist die Typologie der argumentativen Topoi in der Literatur, die seit Aristoteles immer wieder neu versucht wird. Kienpointner (1992: 241f.) referierte die sechs Grund-Klassifizierungen der argumentativen Topoi aus der Rhetorik-Tradition, die dreifach dichotomisch gegliedert sind:

- deskriptive Topoi vs. normative Topoi,

- Pro und Kontra sowie

- reale vs. fiktive Topoi.

Der Argumentierende kann Argumente auf der deskriptiven Basis dessen, was $d a$ ist, produzieren oder auf der Basis seines normativen Wissensbestandes, d.h. auf der Basis dessen, was sein soll. Deskriptive Argumente dienen daher zum Erweis der Wahrheit von strittigen Sachverhalten, während normative Argumente zum Erweis der Richtigkeit von strittigen Sachverhalten dienen (Kienpointner 1992: 241). Ein Beispiel für das deskriptive Argumentationsmuster ist das folgende:

Das Tier ist ein Lebewesen. - Das Pferd ist ein Tier. - Also ist das Pferd auch ein Lebewesen.

Folgendes Beispiel soll das normative Argumentationsmuster verdeutlichen:

Alle Christen sollten ihre Feinde lieben. - Katholiken sind Christen. - Also sollten Katholiken ihre Feinde lieben. ${ }^{1}$

ten explizit genannt und entsprechen somit weitestgehend dem hier präsentierten Argumentationsschema.

1 Beide Beispiele finden sich in den Belegen für die Typologie Kienpointners (1992: 264 f.). 
Reale Argumentationsschemata sind solche, bei denen der Sprecher seine Argumente in Bezug auf die reale Welt produziert und bei denen die Indikativ-Form des Formulierten als Indikator dient, während die fiktiven Argumentationsschemata von der Möglichkeit kontrafaktischer Annahmen ausgehen und das Gesagte im Konjunktiv II formulieren (Kienpointner 1992: 264 f.).

Kienpointner stellt eine spezifische Typologie der Subklassen der argumentativen Topoi in 60 Klassen auf (z. B. das Einordnungs-, Definitions-, Genus-Spezies-, GanzesTeil-, Vergleichs-, Gegensatz-, Kausal-, Analogie- und Autoritätsschema, ebd.: 250-273), die von Ottmers (1996: 86-117) in leicht umsetzbarer Aufstellung präsentiert werden. Dabei handelt es sich ebenfalls um Großklassen von Argumentationsschemata wie das Kausal-, Vergleichs-, Gegensatz- und Einordnungsschema, die von den drei oben genannten Dichotomien abgeleitet sind. Kindt (1992) erweitert diese Schemata noch um einige wichtige weitere wie das Konsequenz- und das Relevanzschema und zeigt durch seine Analyse des Diskurses über den amerikanischen Golf-Krieg, dass man neben den Listen der argumentativen Topoi auch ad hoc neue Topoi einführen kann, um Argumentationstechniken besser beschreiben zu können. Seine Topoi wie der Katastrophen-Topos, der Heile-Welt-Topos, der Zeit-Topos etc. belegen den hier vertretenen Standpunkt von der Themenspezifik der argumentativen Topoi. Auch Wodak (1999) bemühte sich um diese Typologie und erwähnt unter anderem den Topos der Ignoranz, des Unterschiedes, den Topos illustrativer Beispiele. Gleichzeitig zeigen die Ansätze Kindts und Wodaks nebst anderen neueren Ansätzen zur Typologie der argumentativen Topoi, dass »keine einheitliche oder vollständige Typologie alltagsrelevanter Argumentationsmuster existiert «(Hoffmann 2012: 62). Jeder Autor stellt seine eigene Liste und seine Typologie der argumentativen Topoi gemäß seinem jeweiligen Untersuchungsgegenstand auf, wobei die klare Definition des Begriffs argumentativer Topos der gemeinsame Nenner aller Typologien bleibt.

Ottmers (1996) macht in diesem Zusammenhang eine wichtige Unterscheidung zweier Aspekte des Topos in dem hier präsentierten Sinne: Er unterscheidet zwischen dem kontextabstrakten Aspekt eines Topos im engeren Sinne eines formalen Argumentationsschemas und dem kontextrelevanten Aspekt. So ist der Kausal-Topos ein kontextabstrakter Topos und kann für unendlich viele Situationen verwendet werden. Die Füllung dieses kontextabstrakten Topos mit Inhalt in der konkreten Situation macht ihn für die jeweilige Situation erst »kontextrelevant«. Der KausalTopos lässt sich etwa mit folgendem Beispiel kontextrelevant machen und die Kausalität als Ursache und Wirkung konkretisieren: Wenn es regnet, wird die Straße nass. Von besonderer Bedeutung für das Anliegen dieses Beitrags ist ein Hinweis von Ottmers (1996: 90), an den die Argumentationsschulung mit Hilfe der argumentativen Topoi im DaF-Unterricht anknüpfen kann:

»Die kontextrelevanten Topoi beruhen einerseits auf konventionalisierten Schlussverfahren und sind dadurch sehr viel stärker als die kontextabstrakten Topoi den Veränderungen unseres Meinungs- und Erfahrungswissens ausgesetzt.« 
Demzufolge sind abstrakte Topoi wie z. B. der Ursache-Wirkungs-Topos zwar universal, zeitlos und kultur-unabhängig, kontext- und themenabhängige Topoi derselben abstrakten Topoi bleiben jedoch an ihre eigene Zeit, an ihr Thema bzw. an ihre Produktions-Kultur gebunden und sollen zudem »adressatengerecht « produziert und vorgetragen werden. Argumentative Topoi in einem Text zu analysieren bedeutet daher zwangsläufig, zu verstehen, wie der Sprecher seine Ansprechpartner in der Wahl und in der Struktur seiner Argumentationsschemata mit berücksichtigt hat. Argumentative Topoi zu produzieren bedeutet somit, sich themenund publikumsspezifisch zu orientieren. Gerade publikumsspezifische, d.h. kontextrelevante Topoi bzw. Enthymeme produzieren zu lernen, ist das pädagogische Hauptanliegen von Emmel (1994). Heggelund (2001) geht den umgekehrten Weg und plädiert dafür, dass enthymematisches Sprechen gerade dazu besonders geeignet ist, Abtönungspartikeln mit argumentativer Funktion im DaF-Unterricht zu lehren, und führt dafür Beispiele an wie:

Ich bin doch ein ehrlicher Mensch, wozu soll ich Sie denn belügen!

Der Ansatz Heggelunds bestätigt den Ansatz dieses Beitrags, dass argumentatives Sprechen alle Sprachfähigkeiten des Lerners erforderlich macht und den bewussten wahrnehmenden Spracherwerb stimuliert. Dass ohne ausgebaute Sprachkompetenz Argumentationen nicht möglich sind, dürfte als evident gelten. Für Wengeler (1999: 39) reicht schon die Analyse von argumentativen Topoi in geschichtlichen, thematisch abgegrenzten Diskursen im Unterricht als erster Schritt, um Lernern solche Argumentationsschemata beizubringen und sie in die Lage zu versetzen, Sprechen mit Hilfe argumentativer Topoi zu produzieren:

»Das Lernziel bei der Analyse kontextspezifischer Topoi in einem thematisch bestimmten öffentlichen Diskurs ist nicht die manipulationstheoretisch motivierte Entlarvung »falscher « Argumentationen, sondern [...] die Einsicht, dass in bestimmten z. T. über Jahrzehnte verlaufenden politischen Streitfragen [...] mit einem bestimmten Inventar von Argumentationsmustern "gearbeitet « wird und dass die öffentlich Sprechenden auf dieses Inventar angewiesen sind, da sie nicht bei jeglicher Stellungnahme wieder inhaltlich Neues vorbringen können.«

Solche Kenntnisse inhaltlich und thematisch gebundener Topoi, welche die Lerner durch die Analyse argumentativer Texte zu einem bestimmten Thema unter der Leitung der Lehrkraft herausarbeiten, sollen als eine Tür zur Erschließung argumentationsspezifischer Landeskunde in der Zielsprache Deutsch dienen und die Lerner befähigen, mit Vertretern des deutschen Kulturkreises »kulturkonform« zu argumentieren, denn die Deutsch-Lerner

»sollen [...] einschätzen können, wer zu welchen Zeitpunkten auf welche Muster zurückgreift, wie diese also z. T. über Jahre hinweg gleich bleiben, sich z. T. aber auch verändern und wie bestimmte Topoi entweder nur von einer bestimmten Gruppe oder von allen gesellschaftlichen Gruppen verwendet werden oder im Laufe der Zeit 
die Träger der verschiedenen Argumentationsmuster wechseln. Auf der Grundlage dieser Kenntnisse sollen sie sich eine eigene Meinung über die Plausibilität der einzelnen Muster bilden können und sie reflektiert in ihr eigenes Denken und Sprechen über das Thema integrieren oder sie aus diesem ausschließen.« (Wengeler 1999: 39).

Wengeler schreibt diese Zeilen vor dem Hintergrund seiner späteren umfangreichen Monographie (2003), in der er am Beispiel des Einwanderungsdiskurses in Deutschland »themenspezifische und argumentationsträgerspezifische Topoi« nachweisen konnte. Sein Vorschlag, über das Studium langjähriger geschichtlicher Diskurse zu themen- und argumentationsträgerspezifischen Topoi zu kommen, dient primär dazu, die Routine der Reproduktion argumentativer Topoi zu beleuchten, darf aber nicht als »ausschließlich « verstanden werden. Denn die Didaktisierung dieser Topoi lässt sich auch vornehmen, wenn man die Argumentation in bestimmten Kontexten synchron vornimmt (vgl. Kindt 1992: 192), z. B. argumentative Topoi in politischen Wahlkämpfen, in kommerzieller Werbung, zu einer bestimmten Branche der kommerziellen Werbung wie etwa der Kosmetik- oder Automobilindustrie, in Diskursen vor Kriegen, in politischen Gedichten zu einem bestimmten Zeitpunkt usw. All diese Situationen kann der DaF-Unterricht zum Gegenstand machen, um argumentative Topoi der Zielsprache Deutsch zu didaktisieren und den DaF-Lerner mit kulturspezifischen sowie themenspezifischen argumentativen Topoi vertraut zu machen, ohne sich mit jahrzehntelangen Diskursen auseinandersetzen zu müssen. Das hat für die DaF-Lerner den Vorteil zu lernen, welche Argumente und welche argumentative Topoi gerade »aktuell und salonfähig « sind und welche nicht, damit sie sich diese Argumentationsmuster auch zu eigen machen und in ihr Schreiben und Sprechen mit integrieren können. Gerade die synchrone Sichtung der Argumentationsmuster macht es möglich, dass sich DaF-Lerner in relativ kurzer Zeit einen Überblick über thementypische Argumentationsmuster möglichst vieler Themen verschaffen. Eine solche synchrone Bearbeitung der Themenvielfalt macht es erst möglich, dass die DaF-Lerner differenzierte Fertigkeiten bei der Produktion und beim Einsatz von argumentativen Topoi erlangen. Denn der passende argumentative Topos, der kontextrelevant sein soll, dürfte sich, um als »angemessen « gelten zu können, ändern, je nachdem, ob sich die strittigen Sachverhalte in einer kleinen Gruppe oder vor einem großen Publikum, in einer vertrauten oder in einer fremden Umgebung, in einer Herrschaftssituation oder in einer symmetrischen Kommunikationssituation ergeben. Die kontrastive Arbeit von Drewnowska-Vargáné (2001) zu den argumentativen Topoi in argumentativen Leserbriefen in deutscher, polnischer und ungarischer Sprache sowie die Arbeit von Ohlinger (2003) zeigen, dass argumentative Topoi ein großes Potential für die Didaktisierung der argumentativen Kompetenz von DaF-Lernern haben. 


\section{3. Überlegungen zur Didaktisierung argumentativer Topoi im DaF- Unterricht}

Es ist zwar oben erwähnt worden, dass die bewusste Schulung zur Anwendung argumentativer Topoi nach Aristoteles in einer fortgeschrittenen Stufe ab dem Niveau $\mathrm{C} 1$ beginnen sollte. Ich neige jedoch eher dazu, diese Schulung zur Argumentation schon ab dem Niveau B2 zu beginnen, da viele DaF-Lerner nach B2 die DSH-Prüfung ablegen, um z. B. ein Universitätsstudium zu beginnen, wo besonders die argumentative Kompetenz in den unterschiedlichen Disziplinen des Studiums und des wissenschaftlichen Schreibens verlangt wird. Es dürfte zudem selbstverständlich sein, dass die Qualifizierung zur argumentativen Kompetenz schon mit der ersten Stunde im DaF-Unterricht beginnt, denn die argumentative Kompetenz ist ja die Krönung und zugleich die Messlatte der bisherigen linguistischen, kommunikativen und interkulturellen Fertigkeiten sowie des Weltwissens.

Den Lernern Topoi näherzubringen kann selbstverständlich durch die Analyse von schriftlichen Texten und mündlichen Sprachzeugnissen erfolgen, wie bereits oben am Ansatz von Wengeler angedeutet wurde. Möglichkeiten und Situationen, in denen DaF-Lerner selbst argumentative Topoi bzw. Argumente finden und verwenden, gibt es unendlich viele. Wichtigstes Merkmal des sprachlichen Materials in deutscher Sprache muss die oben angesprochene argumentative Textentfaltung sein, bei der Thesen aufgeworfen und begründet oder widerlegt werden oder für bzw. gegen bestimmte Positionen geworben und plädiert wird. Solche argumentative Textentfaltung findet man etwa in der schöngeistigen Literatur, z. B. in politischen Gedichten, Zeitungskommentaren und in weiteren Zeitungstexten, in Briefen, Leserkommentaren, Rechtstexten, in politischer und kommerzieller Werbung, um nur einige prominente Beispiele zu nennen. Die Texte und Situationen sollen möglichst lebensnah und realistisch sein, damit sich die DaF-Lerner für die in diesen Sprachzeugnissen vertretenen Standpunkte auch wirklich interessieren. Auch lässt sich der Einsatz verschiedener Arbeitsformen und verschiedener Medien in Erwägung ziehen, um den Lernern einen eher »kopflastigen«, trockenen Lernstoff interessanter zu machen, wie unten darzulegen ist.

Eine ausgewogene, zielgerichtete, eigene bewusste Didaktisierung argumentativer Topoi im DaF-Unterricht muss zwei Ziele erfüllen: den Lernenden die Wirkung und die Realisierungsformen argumentativer Topoi in deutschsprachigen Texten und Gesprächen näher zu bringen und sie sukzessive in die Lage zu versetzen, selbst Texte und argumentative Gespräche zu produzieren, in denen sie selbst solche Topoi verwenden, wobei der Hinweis, wie bereits oben gesagt, nicht fehlen darf, dass die Beherrschung argumentativer Topoi nur einen Teil der Fertigkeiten darstellt, welche für die Befähigung zur flüssigen, verständigungsorientierten Argumentation erforderlich sind. 
Ein weiterer wichtiger Punkt bei der Didaktisierung ist die Tatsache, dass das Ziel einer solchen Integration argumentativer Topoi in die Argumentationsschulung im DaF-Unterricht nicht nur ist, sich mit einem erwartbaren thementypischen bzw. kulturkreistypischen Katalog von argumentativen Topoi vertraut zu machen, sondern den Prozess der Produktion und Verwendung von Topoi zu beherrschen. Denn der Katalog der in einem Diskurs enthaltenen argumentativen Topoi ist immer das Ergebnis einer Analyse des jeweiligen Textes oder der jeweiligen Texte.

Die oben genannte Definition des Topos nebst den einschlägigen Typologisierungen der Topoi wird sicherlich im Unterricht wegweisend sein.

Wie werden die Lernenden aber herausfinden können, ob die Topoi in den analysierten Texten auch tatsächlich »argumentationswirksam « sind oder nicht? Wie können die DaF-Lerner sicherstellen, ob die argumentativen Topoi, die in ihren Texten oder ihren Gesprächsbeiträgen enthalten sind, tatsächlich den jeweiligen Opponenten überzeugen? Wie können die DaF-Lerner den Standpunkt des Opponenten im Licht der zum Zuge gekommenen argumentativen Topoi widerlegen, wenn der Standpunkt zu widerlegen ist?

Um die DaF-Lerner in die Lage zu versetzen, all das zu tun, sind gewisse übersichtliche Kriterien erforderlich, die ihnen in der ersten Phase helfen, die Arbeit mit argumentativen Topoi in Analyse und Produktion schrittweise zu erlernen, bis die Stufe der Spontaneität erreicht ist. Ein Blick auf die altgriechische RhetorikSchulung, insbesondere auf das für europäische Bildung einflussreiche altgriechische Rhetorik-Übungsbuch Progymnasmata (Kennedy 2003), könnte hier Abhilfe schaffen (vgl. auch Winkler 2003: 179 f.). Der Name Progymnasmata bedeutet auf Deutsch »Vor-Übungen« (vgl. Ueding/Steinbrink 1994: 181, 331). Ähnlich wie die Körperübungen sollte der Redner auch in der freien argumentativen Rede VorÜbungen absolvieren, um sich als fähiger Redner in der konkreten Diskussion behaupten zu können. Eine der wichtigsten Übungen in diesem Buch ist die Schulung zur Bekräftigung (confirmation) und Widerlegung (refutation) von Standpunkten, die zu insgesamt 14 Übungen gehört, die auf den griechischen Rhetoriker Aphthonios von Antiochia zurückgehen sollen. Confirmation und Refutation gelten - neben diesem Verständnis als Progymnasmata - als genuine Teile der klassischen Rede, der partes orationis.

Sowohl bei der Bekräftigung eines Standpunktes, sei dies der eigene oder der Standpunkt anderer, als auch bei der Widerlegung bieten sich hier klare Anweisungen, wie man mit implizierten Argumentationsschemata umgeht bzw. wie man die vorgebrachten Argumente und die sie stützenden deduktiven Schlussmuster überprüfen kann.

Zur Vorgehensweise bei der Widerlegung lesen wir in Progymnasmata: 
»Those engaged in refutation should first state the false claim of those who advance it, then add an exposition of the subject and use these headings: first, that it is unclear and incredible, in addition that it is impossible and illogical and inappropriate, and finally adding that it is inexpedient." (Kennedy 2003: 101)

Zur Vorgehensweise bei der Verteidigung bzw. Bekräftigung eines Standpunktes ist, ebenfalls in diesen »Vor-Übungen«, zu lesen:

»One who is confirming would use arguments opposed to those of refutation and first mention the good repute of the claimant, then, in turn, provide an exposition, and use the opposite headings: clear instead of unclear, credible instead of incredible, and possible instead of impossible and logical instead of illogical and appropriate instead of inappropriate and expedient instead of inexpedient.« (ebd.)

Die Anweisungen für die Verteidigung und Widerlegung von Standpunkten lassen sich wie folgt wiedergeben:

Bei der Verteidigung/Bekräftigung bzw. Stützung/Vertretung von Standpunkten in argumentativen Diskursen soll der DaF-Lerner folgende Schritte befolgen:

1. Lob für die Integrität/Glaubwürdigkeit der Person, die den Standpunkt geäußert hat

2. Erstellung einer kurzen Wiedergabe des Standpunktes

3. Darlegung, warum die verwendeten Argumente zum vertretenen Standpunkt Anspruch auf folgende Attribute erheben:
A) verständlich
B) glaubwürdig
C) möglich
D) logisch
E) angemessen
F) zweckmäßig

Bei der Widerlegung/Anfechtung von Standpunkten in argumentativen Diskursen soll der DaF-Lerner hingegen folgende Schritte befolgen:

1. Zurückweisung der Behauptung derjenigen Person, die die Behauptung aufgestellt hat

2. Erstellung einer kurzen Wiedergabe dieser Behauptung

3. Darlegung, warum die verwendeten Argumente zum vertretenen Standpunkt, mit folgenden Attributen beschrieben werden können:
A) un-verständlich
B) un-glaubwürdig
C) un-möglich
D) un-logisch
E) un-angemessen
F) un-zweckmäßig. 
Die Alltagsargumentation, wie wir sie heute kennen, hält sich nicht immer an die ersten beiden Schulungs-Schritte sowohl bei der Verteidigung als auch bei der Widerlegung. Nach der aufmerksamen Beobachtung dieses Autors werden die ersten beiden Schritte am häufigsten nur dort beachtet, wo die Argumentation schriftlich realisiert wird und an den Argumentierenden höhere intellektuelle Ansprüche gestellt werden. In der Alltagsargumentation schreitet man in der Regel direkt zur Bekräftigung bzw. Anfechtung des Standpunktes mit einer oder mehreren dieser sechs Kategorien und setzt einfach die kontextuelle Vertrautheit der Angesprochenen mit dem Sachverhalt voraus. Diese sechs Kategorien zur Verteidigung der Argumente und ihre Gegenteile zur Widerlegung sind zudem natürlich etwas anderes als die Typologie der argumentativen Topoi, die oben referiert worden ist. So kann z. B. ein Kausalitätstopos logisch oder unlogisch sein.

Eine Familie mit 7 Personen, die vorrangig ein Auto mit ausreichenden Sitzplätzen für alle Familienmitglieder kaufen will, wird folgenden Kausalitätstopos benutzen, weil es logisch ist:

Wir kaufen dieses Auto, weil es sieben Sitze hat.

Wenn aber dieselbe Familie folgenden Kausalitätstopos benutzt,

Wir kaufen dieses Auto, weil es blau ist.

dann ist der Topos zwar ein Kausalitätstopos, aber prioritäts- und kontextbedingt unlogisch, unangemessen, unverständlich und unzweckmäßig.

Daher stellen diese sechs Kategorien und ihre Gegenteile ein zusätzliches wichtiges Instrumentarium bei der Didaktisierung der argumentativen Topoi im DaFUnterricht dar sowohl in der Analyse als auch in den Übungen zur Erstellung von Texten mit solchen Argumentationsmustern, da sie wie eine Art Bewertungsraster fungieren.

Interessant an diesen Kategorien ist zudem die Tatsache, dass ihre Handhabung in der kulturspezifischen Schulung der Argumentation im DaF-Unterricht zum größten Teil auf fundierten Kenntnissen der deutschen Sprache und der deutschen Zielkultur beruht. Denn ohne fortgeschrittene deutsche Sprachkenntnisse ist eine verständliche Formulierung von Argumenten in Texten und Gesprächen nicht möglich. Auch die Attribute der »Angemessenheit«, der »Glaubwürdigkeit« und der »Möglichkeit« von Argumenten und impliziten Schlussmustern setzen die genaue Kenntnis des Zielpublikums und der Besonderheit des argumentativen Kontextes voraus. Insofern stellt der Einsatz der argumentativen Topoi durch den DaF-Lerner einen kulturell intensiven Dialog mit der deutschen Zielkultur dar.

Nun bleibt die Frage, welche dieser sechs Kategorien die Priorität vor den anderen hat und ob man eine solche angegebene Reihenfolge strikt befolgen muss. Corbett 
und Connors (1999) machen sich Gedanken über diese Frage im Zusammenhang mit der Confirmation und kommen zum Schluss, dass man zuerst mit einem starken Argument beginnen soll und dann die weniger starken Argumente folgen lassen soll, um schließlich mit dem stärksten Argument zu enden (Corbett/Connors 1999: 277 f.). Offensichtlich bleibt dies ebenfalls von der jeweiligen Situation abhängig, welche der genannten Attribute vorrangig thematisiert werden sollen, auch wenn anzunehmen ist, dass das Logische bei allen Argumentationen die maßgebliche Rolle spielt.

Bei der Widerlegung (Refutation) konkretisieren Corbett und Connors ebenfalls, dass dies (die Widerlegung) durch eine der beiden Optionen möglich ist: entweder die Wahrhaftigkeit der Prämisse zurückzuweisen, auf die die Konklusion zurückgeht, oder die Richtigkeit der Schlussfolgerung der Konklusion aus der Prämisse zurückzuweisen und als unmöglich zu bezeichnen (a. a. O.: 279). Bei dieser Vorgehensweise der Widerlegung spielt das Logische als eines der sechs Attribute, die im Buch der Progymnasmata vorkommen, die dominante Rolle. Das heißt, hier wird ausschließlich auf der logischen Schiene zu widerlegen versucht. Die Autoren raten (Corbett/Connors 1999: 31-84; 279-283) daher - unter Rückgriff auf die drei Arten der Überzeugung bei Aristoteles: logos (logische Beweisführung), pathos (emotionaler Appell) und ethos (Autorität und Glaubwürdigkeit des Sprechers) -, den Kontrahenten in seiner menschlichen Ganzheit anzusprechen, was durch den Einsatz der Strategien der Emotionalisierung und der vom Sprecher repräsentierten ethischen Werte möglich wird. Bei der Emotionalisierung geht es um einen emotional gesättigten Appell, der genau auf die Besonderheiten der Zielgruppe zugeschnitten sein soll. Bei den ethischen Werten geht es um diejenigen Werte, die sich der Sprecher zuschreibt und damit sein Charisma und sein Image in der Argumentation zur Geltung bringt. Erst der Rückgriff auf die Emotionalisierungsstrategien und auf allseits akzeptierte ethische Werte kann dem logischen Argument zum Erfolg verhelfen. Denn selbst wenn ein logisches Argument stimmt, kann es immer noch auf Ablehnung stoßen, wenn sich die Angesprochenen emotional gegen diese Argumente oder gegen die Person sträuben, die diese Argumente äußert. Das ist ein weiterer Beleg dafür, dass sich die Schulung zur Argumentation im DaF-Unterricht, insbesondere zur Verwendung und zum Einsatz argumentativer Topoi, die Kenntnis des jeweiligen konkreten deutschen bzw. des deutschsprachigen Publikums zwingend voraussetzt. Die Schulung zum Einsatz argumentativer Topoi trägt auch zur Schulung im Bereich der interkulturellen Kommunikation bei. 


\section{Zur didaktischen Umsetzung der argumentativen Topoi im DaF- Unterricht}

\subsection{Der Einstieg}

Ziele dieses Einstiegs sollen sein, dass die DaF-Lerner als Basis erklärt bekommen

- was argumentative Topoi eigentlich sind

- was ein Enthymem in der altgriechischen Tradition und in der heutigen Verwendung bedeutet und wann Enthymem als »verkürzter Syllogismus« bezeichnet wird

- wie diese rhetorischen Begriffe voneinander zu unterscheiden sind

- wie diese rhetorischen Strukturen in der natürlichen Sprache bzw. in der Alltagsargumentation realisiert werden (können).

Wichtigstes Instrument der DaF-Lehrkraft bei dieser Einstiegs-Phase ist, dass sie über klare Definitionen dieser Begriffe mit guten sprechenden Beispielen verfügt, die auch separate Einzelsätze sein können.

Dabei gehört die Erklärung der Typologien der argumentativen Topoi meines Erachtens nicht zu diesem Einstieg. Der Einstieg soll nicht zu einer »trockenen Rhetorik-Stunde« mutieren, bei der die DaF-Lerner den Sinn des Unterrichts aus den Augen verlieren. Daher darf nicht mit diesen Begriffen angefangen werden, sondern man geht von den natürlichsprachlichen Belegen aus, welche von den DaFLernern unter der Leitung der jeweiligen DaF-Lehrkraft gemeinsam betrachtet werden. Der häufigste Argumentationstopos ist nach vielen Autoren (Kienpointner 1992: 328; Hoffmann 2012: 219) im Lichte der praktischen Analyse des Sprachmaterials der Topos der Kausalität. Zugleich lässt sich die sprachliche Realisierung dieses argumentativen Topos gut mit Konjunktoren, Subjunktoren sowie mit einigen Abtönungspartikeln, wie oben bereits angedeutet, verbinden. Solche sprachlichen Realisierungen argumentativer Topoi können dazu beitragen, neue Einsichten in die pragmatischen Funktionen vertrauter sprachlicher Strukturen der Zielsprache Deutsch zu gewinnen. Beispiele zur Betrachtung und zur Diskussion in der Klasse, an die die Erklärung der rhetorischen Begriffe anknüpfen kann, wären für die kausalen Topoi etwa:

A) Du wirst im Studium Erfolg haben, denn du arbeitest fleißig.

B) Weil die Rechtssicherheit in Afrika fragwürdig ist, wollen die Investoren dort nicht investieren.

C) Da er zu spät aufgestanden ist, konnte er den Zug nicht erreichen.

D) Er ist heute ein kluger Geschäftsmann, da er ja oft Schaden erlitten hatte.

Diese Beispiele sind meines Erachtens geeignet, um einige Facetten der Kausalitäts-Schemata zu erklären und die DaF-Lerner in die Thematik der argumentati- 
ven Topoi einzuführen. In all diesen Beispielen ist die Schlussregel implizit und lässt sich leicht in einen vollständigen Syllogismus umwandeln. Während im Beispiel A) von der »Wirkung « auf die »Ursache « geschlossen wird, wird in Beispiel B) von der Ursache auf die Wirkung geschlossen. Beispiel C) zeigt, dass ein Ausbleiben der Ursache zum Ausbleiben der erhofften Wirkung geführt hat. Im Beispiel D) wird im normativen Sinne zuerst die Wirkung angeführt, gefolgt von der Ursache.

Auf diese Weise lernen die DaF-Lerner bewusst an ersten Beispielen, wie Kausalität und kausale Argumentationsmuster in der deutschen Sprache realisiert werden können. Auch durch diese Einzelsätze können die DaF-Lerner selbst feststellen, ob diese Argumente verständlich oder unverständlich, logisch oder unlogisch, angemessen oder unangemessen, zweckmäßig oder unzweckmäßig, möglich oder unmöglich sind. Denn die Gegenüberstellung des Satzes

Du wirst im Studium Erfolg haben, denn du arbeitest fleißig.

mit dem Satz

Du wirst im Studium Erfolg haben, denn du hast blaue Augen.

verhilft den Lernern zur eigenen Wahrnehmung der sechs Attribute der Argumente, wie sie in Progymnasmata vorgeschlagen werden. Die DaF-Lernenden werden selbst keine evidente logische Kohärenz zwischen dem Erfolg im Studium und den blauen Augen feststellen können, wie dies der Fall zwischen dem Studienerfolg und dem Fleiß ist. Daher erscheint das letzte Beispiel als unlogisch, unverständlich und unangemessen. Dass diese Gegenüberstellung eine positive Wirkung auf die Fähigkeiten der DaF-Lernenden bei der Analyse und Produktion argumentativer Topoi haben wird, dürfte auf der Hand liegen.

\subsection{Grass-Gedicht als praktisches Beispiel für mündliche und schriftliche Übungen}

Erst nach dem gelungenen Einstieg ins Thema mit Hilfe der Betrachtung und der Diskussion von guten Beispielen kann der DaF-Unterricht zur Analyse und zur mündlichen und schriftlichen Übung der argumentativen Topoi übergehen.

Vorrangige Ziele dieser Analyse-Übung, ob mündlich oder schriftlich, sollen sein:

- Erkennen der sprachlichen Realisierungsmöglichkeiten von argumentativen Topoi in authentischen Texten und Kontexten,

- Erkennen, wie man mündlich wie schriftlich mit Hilfe argumentativer Topoi antizipative Argumente in seine Rede oder den eigenen Text einbauen kann, 
- Erlangung der Fertigkeit, einen Standpunkt zu befürworten, zu bekräftigen und mit guten Argumenten zu unterstützen (Confirmation),

- Erlangung der Fertigkeit, einen Standpunkt zu widerlegen bzw. die verwendeten Argumente in Frage zu stellen (Refutation),

- Erkennen, dass man mit argumentativen Topoi bzw. mit ein und demselben argumentativen Topos unterschiedliche bzw. gegensätzliche Standpunkte vertreten kann,

- Erlangung der Fertigkeit, nur relevante Informationen für die jeweiligen Argumente zu wählen und die Konklusionen auf die Prämissen abzustimmen.

Die Vorgehensweise soll hier an einem Beispiel gezeigt werden. Dieses Beispiel umfasst

A) Verse des argumentativ ausgerichteten politischen Gedichts von Günter Grass »Was gesagt werden muss« als Primär-Text

B) Beispiele von Online-Kommentaren zu diesem Gedicht als Sekundär-Texte, welche sich wiederum in Zustimmung/Bekräftigung (also Confirmation) und Widerlegung (Refutation) der Standpunkte im Primärtext teilen.

Das Gedicht ist erstmals am 4. April 2012 in der Süddeutschen Zeitung erschienen ${ }^{1}$, fand eine rasante Weiterverbreitung in anderen deutschen Medien und nur wenige Tage später in internationalen Medien als Übersetzung (z. B. auf Hebräisch, Persisch, Arabisch und Englisch). In den Online-Kommentaren finden sich ebenfalls auf der Webseite der Süddeutschen Zeitung im Anschluss an das Gedicht 240 Leserkommentare. Grass vertritt darin mehrere Standpunkte und begründet den jeweiligen Standpunkt auch mit argumentativen Topoi.

Im Gedicht plädiert der Autor dafür, keine U-Boote an Israel zu exportieren und Israel und den Iran unter internationale Kontrolle zu stellen. Es sollen in diesem sehr engen Rahmen keine ganzheitliche literarische oder politische Analyse des Gedichts mit präsentiert, sondern nur praktische didaktische Vorschläge zur Verwendung des Gedichts im DaF-Unterricht gemacht werden, um die DaF-Lernenden mit dem Phänomen der argumentativen Topoi vertraut zu machen. Das Gedicht ist meines Erachtens besonders geeignet für den Einsatz im DaF-Unterricht mit dem Ziel der Didaktisierung der Argumentationsmuster, und zwar aus folgenden Gründen, die auch bei der Auswahl geeigneter Texte hilfreich sein können:

1 Siehe unter folgendem Link: http://www.sueddeutsche.de/kultur/gedicht-zum-konfliktzwischen-israel-und-iran-was-gesagt-werden-muss-1.1325809? commentspage=all:7: \#comments: abgerufen am 30.12.2013. 
- Es ist aktuell, transferiert aktuelles Wissen zur deutschen Landeskunde und ist von einem aktuellen großen deutschen Schriftsteller geschrieben.

- Es ist argumentativ, bzw. im Stil der Alltagsargumentation aufgebaut und transferiert »schnelles« Wissen zu den argumentativen Topoi zu einem bestimmten Thema, nämlich zur Verantwortung der Deutschen vor ihrer Geschichte und den deutsch-israelischen Beziehungen sowie zum Beitrag Deutschlands zu Weltfragen wie Krieg und Frieden.

- Die argumentativ gestalteten Online-Kommentare eignen sich hervorragend zur Ein- und Ausübung der beiden Argumentationsformen Confirmation und Refutation, da die DaF-Lerner in diesen Online-Kommentaren bereits vorausgehende authentische Beiträge vorfinden.

- Das Gedicht richtet sich an die eigene deutschsprachige Gemeinschaft (Bevölkerung und Regierung), an Israel, an den Iran und an die Weltbevölkerung, für die das Thema Krieg und Frieden relevant ist. Die argumentativen Topoi sind zum Teil »kontextrelevant«, kulturgemeinschaftstypisch und universeller Natur, da hier ein heterogenes Publikum angesprochen wird.

Dabei bieten sich zwei Vorgehensweisen an:
A) die schriftliche und
B) die mündliche Art.

In beiden Fällen ist eine Phase der Vorarbeit erforderlich. Diese Vorarbeit soll zum Thema der argumentativen Topoi hinführen und stellt das Eingeständnis dar, dass sich der moderne integrative DaF-Unterricht nicht mit Einzelerscheinungen des Sprachgebrauchs befassen kann, ohne die damit im Zusammenhang stehenden Phänomene zu berücksichtigen. Diese Vorarbeit umfasst:

A) die Erstellung eines (bilingualen) Glossars mit den schwierigen Wörtern des Gedichtes. Dabei bedienen sich die DaF-Lerner zu dieser Vorbereitungsaufgabe neben der selbständigen Erschließung der Bedeutung aus dem Kontext - zweisprachiger Wörterbücher (Deutsch-Englisch, Deutsch-Arabisch usw.). Zur Vorbereitung gehört auch das selbständige erste Lesen des Gedichtes durch jeden Kursteilnehmer;

B) die gemeinsame Lektüre des Gedichtes und die Präsentation der Inhaltsangaben durch die DaF-Lernenden. Diese Inhaltsangaben sollen zunächst mündlich vorgetragen und dann als schriftliche Hausaufgabe angefertigt werden, wobei die DaF-Lernenden bewusster die eingesetzten Argumente wahrnehmen können. Solche Inhaltsangaben sind zwar äußerlich ein Vorgriff auf die schriftliche Einübung der argumentativen Topoi, sie verfolgen jedoch als klassische Aufgabe im Sprachunterricht ausschließlich das Ziel des ersten Sich-Informierens durch die 
zusammenfassende Wiedergabe des Originaltextes (vgl. Reinert 2012: 475) und nicht vorrangig die zu erlernende Erschließung der expliziten und impliziten Argumentationsstrukturen;

C) die Präsentation des Gedicht-Kontextes sowie kurzer Informationen zum Leben und Werk des Dichters im Plenum.

Mit diesen drei Vorbereitungsschritten gilt die Vorarbeit der Argumentations- und Topos-Schulung als abgeschlossen. Danach geht es darum, das Gedicht in Bezug auf die Topoi und die Argumente, die darin vorkommen, zu analysieren - auch mit dem Ziel, einen Katalog der argumentativen Topoi zu erstellen, die der Dichter verwendet hat. Es bieten sich hier die rein schriftliche und die gemischt mündlichschriftliche Vorgehensweise an.

A) Bei der mündlich-schriftlichen Vorgehensweise kann der Kursleiter die Lerner in Gruppen einteilen, die jeweils einen Teil des Gedichts in Bezug auf die Standpunkte (Konklusionen / die Thesen) und die dazu vom Dichter vorgebrachten Argumente analysieren und diese Argumente auf die implizierten Topoi zurückführen, wobei hier ein Rückgriff auf eine Rollenverteilung aus dem deutschen politischen Leben vorgeschlagen wird.

\section{Das Rollenspiel: Das Bundestagsmodell}

Nach dem Vorbild der Redner im Bundestag können sich die DaF-Lernenden bei dieser schriftlich-mündlichen Vorgehensweise in drei Fraktionen aufteilen:

- eine Fraktion repräsentiert den Dichter als Vertreter Deutschlands bzw. der Deutschen,

- eine Fraktion vertritt die Iraner als Betroffene,

- und eine weitere Fraktion repräsentiert die Israelis als (indirekt) Angesprochene, denen die Planung atomarer Angriffe auf den Iran unterstellt wird.

Aufgabe jeder Fraktion soll sein:

1. Gemeinsame Formulierung der eigenen Behauptung / der eigenen These / des eigenen Stand punktes, um Feedback innerhalb der eigenen Gruppe zu bekommen und sich dadurch kognitiv und argumentativ zu entwickeln;

2. Formulierung der Argumente für die Bekräftigung des eigenen Standpunkts, bzw. ausdrückliche Benennung der verwendeten Topoi;

3. Formulierung eines antizipativen Argumentes zum Schutz des eigenen Standpunkts gegen die potentielle Widerlegung. Hier wird ein negatives Feedback der Kontrahenten einkalkuliert; 
4. Jede Fraktion wiederum hat sich für eine der beiden übrigen Fraktionen zu entscheiden, deren Standpunkt sie mündlich und im Plenum bekräftigen / befürworten und für eine zweite Fraktion, deren Standpunkt sie widerlegen soll. Dabei sollen die implizierten argumentativen Topoi in beiden Fällen ausdrücklich genannt werden. Auf diese Weise bekommt jede Gruppe ein zweifaches Feedback von der ganzen Klasse. Das letzte Feedback für die Leistung jeder Fraktion soll natürlich von der Lehrkraft stammen.

Auf diese Weise bekommen alle mitwirkenden DaF-Lernenden das Selbstfeedback, das zweifache Feedback der Klasse (Confirmation und Refutation) und das Feedback der Lehrkraft, was zum Lernprozess immens beitragen soll (Keller 2012: 135).

Wichtig bei der Behandlung dieses Textes im Unterricht ist die Gewährung von Freiheit bei der Formulierung der hier begründeten Thesen des Gedichts, da ein Gedicht, abhängig von der Perspektive und den Hintergründen des Interpreten, vielfältig ausgelegt werden kann. Schließlich können die DaF-Lerner sehen, dass man eine Sache aus sehr unterschiedlichen Perspektiven mit argumentativen Topoi darstellen kann. Bei der Confirmation und Refutation können die Online-Kommentare zum Gedicht für die DaF-Lerner im Unterricht eine anregende Hilfe sein. Das soll hier an zwei der insgesamt 9 Strophen des Gedichts aufgezeigt werden: Im ganzen Gedicht ist der Kausalitäts- und Konsequenztopos ${ }^{1}$ dominant und daher werden vier Strophen mit »warum«, »Doch warum«, »warum aber«, »warum « und zwei Strophen mit »Jetzt aber, weil...« und »Und zugegeben: ich schweige nicht mehr, weil ..." eingeleitet. Die übrigen Strophen führen Argumente an, welche die »warum-Fragen « ohne »weil« beantworten oder rhetorische Fragen, die Zustimmung erheischen sollen. Der Kausalitätstopos enthält eine UrsacheWirkungs-Relation, während der Konsequenzen-Topos diese Ursache-Wirkungs-Relation um die Folgen im Sinne von negativen und positiven Konsequenzen einer Handlung bzw. einer Unterlassung erweitert (vgl. Kienpointner 1992: 340; Hoffmann 2012: 83). Diese argumentativen Topoi der Kausalität und der Konsequenz können durch die Gruppe der DaF-Lernenden der ersten Fraktion bearbeitet und vorgetragen werden. In der ersten und zweiten Strophe führt der Dichter die Gründe an, warum er endlich das Wort ergreift und das Schweigen bricht:

1 Natürlich enthält das Gedicht mehrere andere Topoi, die für die Argumentation konstituierend sind, wie die Geschichts-, Antisemitismus-, Analogie-, Gute-Moral-, Friedensverantwortungstopoi. In diesem Kontext gelten aber alle hier aufgeführten als Sub-Topoi. Denn es wird hier hauptsächlich begründet, gerechtfertigt, wie es in der Alltagsargumentation üblich ist. 


\section{Strophe}

1. Warum schweige ich, verschweige zu lange,

2. was offensichtlich ist und in Planspielen

3. geübt wurde, an deren Ende als Überlebende

4. wir allenfalls Fußnoten sind.

\section{Strophe}

5. Es ist das behauptete Recht auf den Erstschlag,

6. der das von einem Maulhelden unterjochte

7. und zum organisierten Jubel gelenkte

8. iranische Volk auslöschen könnte,

9. weil in dessen Machtbereich der Bau

10. einer Atombombe vermutet wird.

Der Einfachheit und der Zweckmäßigkeit halber wird diese DaF-Gruppe, wie auch alle anderen, beauftragt, den argumentativ strukturierten Inhalt der beiden Strophen in Prosa-Textform wiederzugeben, was in Teilen einen Rückgriff auf die Vorbereitungsphase darstellt. Das sähe konkret etwa so aus:

1. Der Autor erhebt mit einer rhetorischen Frage den Anspruch darauf, nicht mehr zu schweigen und Klartext zu reden, da die Gefahr offensichtlich ist und bereits in Planspielen geübt wird. Am Ende dieser Planspiele sind wir als Überlebende lediglich wie Fußnoten, falls wir überhaupt überleben sollten.

2. Diese Gefahr besteht darin, dass Israel das Recht auf einen atomaren Erstschlag gegen den Iran reklamiert, und dies allein aus dem Grund, dass lediglich vermutet wird, dass der Iran eine Atombombe besitzt.

3. Dieser Erstschlag könnte das iranische Volk komplett auslöschen.

4. Das iranische Volk ist ohnehin nicht frei und wird vom eigenen Herrscher zum organisierten Jubel gezwungen, der lediglich als »Maulheld «betrachtet werden kann.

Die in diesen Strophen implizierten Topoi können - auch unter der Einbeziehung muttersprachlicher Argumentationskompetenz (vgl. Keller 2012: 129), wie folgt expliziert werden, wobei beachtet werden soll, dass auch mehrfache Zuordnung und mehrfache Klassifizierung der argumentativen Topoi möglich sind:

\section{(A)}

\begin{tabular}{|l|l|}
\hline Prämisse & Günter Grass sieht eine offensichtliche Gefahr. \\
\hline $\begin{array}{l}\text { Schlussregel/argu- } \\
\text { mentativer Topos }\end{array}$ & $\begin{array}{l}\text { Wenn man eine offensichtliche Gefahr sieht, hat man das Recht, } \\
\text { sein Schweigen zu brechen. }\end{array}$ \\
\hline \multicolumn{2}{|c|}{ Topos der offensichtlichen Gefahr (bzw. der Konsequenz) } \\
\hline Konklusion & Günter Grass hat das Recht, sein Schweigen zu brechen. \\
\hline
\end{tabular}


(B)

\begin{tabular}{|l|l|}
\hline Prämisse & $\begin{array}{l}\text { Die Gefahr des atomaren Erstschlags gegen den Iran wird in } \\
\text { Planspielen geübt, an deren Ende wir als Überlebende nur Fuß- } \\
\text { noten sind. }\end{array}$ \\
\hline $\begin{array}{l}\text { Schlussregel/argu- } \\
\text { mentativer Topos }\end{array}$ & $\begin{array}{l}\text { Wenn eine Gefahr in Planspielen geübt wird, an deren Ende wir } \\
\text { als Überlebende nur Fußnoten }{ }^{1} \text { sind, hat man das Recht, sein } \\
\text { Schweigen zu brechen. }\end{array}$ \\
\hline \multicolumn{1}{|c|}{ Schadens-Topos } \\
\hline Konklusion & $\begin{array}{l}\text { Günter Grass hat das Recht, sein Schweigen (zur Schadensver- } \\
\text { meidung) zu brechen. }\end{array}$ \\
\hline
\end{tabular}

(C)

\begin{tabular}{|l|l|}
\hline Prämisse & $\begin{array}{l}\text { Israel droht dem Iran mit einem atomaren Erstschlag auf der Ba- } \\
\text { sis reiner Vermutung, der das ganze iranische Volk auslöschen } \\
\text { könnte. }\end{array}$ \\
\hline $\begin{array}{l}\text { Schlussregel/argu- } \\
\text { mentativer Topos }\end{array}$ & $\begin{array}{l}\text { Wenn eine Atommacht aufder Basis reiner Vermutung mit einem } \\
\text { atomaren Erstschlag gegen ein anderes Land droht, der das ganze } \\
\text { Volk auslöschen könnte, hat man Recht, sein Schweigen zu bre- } \\
\text { chen. }\end{array}$ \\
\hline \multicolumn{1}{|c|}{ Topos der Konsequenz } \\
\hline Konklusion & Günter Grass hat Recht, sein Schweigen zu brechen. \\
\hline
\end{tabular}

${ }^{1}$ Der inzwischen verstorbene Herausgeber der FAZ Schirrmacher weist in einem Leitartikel unter dem Titel »Eine Erläuterung - Was Grass uns sagen will« am 4.4.2012 darauf hin, dass der Ausdruck »Fußnoten der Geschichte« im Gedicht von Grass eine Anspielung auf die Rede der ehemaligen Vorsitzenden des Zentralrates der Juden in Deutschland ist, die sie am 8. November 2008 anlässlich einer Gedenkveranstaltung zur Pogromnacht gehalten hatte und in der sie die Opfer des Holocaust als »Fußnoten der Geschichte« bezeichnete. Das heißt, hier verwendet der Autor denselben Schadenstopos bzw. den Analogie-Topos und sogar denselben Ausdruck, den seine Opponenten, die Israelis und deren Befürworter, verwendet haben. Das ist ein Beispiel dafür, wie die Gedankenwelt und die Denkmuster der angesprochenen Opponenten bei dem Einsatz von argumentativen Topoi berücksichtigt werden sollten, um erfolgreicher argumentieren zu können. Vgl. unter http://www.faz.net/aktuell/feuilleton/debatten/das-israel-gedicht-von-grass/eine-erlaeuterung-was-grass-uns-sagen-will-11708120.html (30.12.2013). 
(D)

\begin{tabular}{|l|l|}
\hline Prämisse & $\begin{array}{l}\text { Das iranische Volk ist unterjocht und zum organisierten Jubel ge- } \\
\text { zwungen. }\end{array}$ \\
\hline $\begin{array}{l}\text { Schlussregel/argu- } \\
\text { mentativer Topos }\end{array}$ & $\begin{array}{l}\text { Wenn ein Volk unterjocht und zum organisierten Jubel gezwun- } \\
\text { gen wird, dann sollte man es unterstützen und es nicht mit einem } \\
\text { Atomschlag auslöschen. }\end{array}$ \\
\hline Konklusion & $\begin{array}{l}\text { Man solite das iranische Volk unterstützen und es nicht mit ei- } \\
\text { nem atomaren Schlag auslöschen (lassen). }\end{array}$ \\
\hline
\end{tabular}

Nun kann die zweite Fraktion diese Argumentation bekräftigen (Confirmation) und zeigen, warum der Autor bzw. die erste Fraktion mit diesen argumentativen Topoi Recht hat. Die Praxis dieser Bekräftigung zeigt unendlich viele Formen, die von der Kurzformel »ja, der Autor hat Recht« bis zur detaillierten begründenden Zustimmung mit Hilfe der sechs Attribute der guten Argumente reicht. Im Folgenden führe ich ein Beispiel aus den Online-Kommentaren an, das als eine Aussage der zweiten Fraktion bei der Confirmation hätte stehen können:

Es findet sich in meinem Korpus folgender Online-Kommentar:

\section{sir-dave 4.4.2012 | 12:55 Uhr}

Auch ich will nicht schweigen......denn zugegeben: Eine Bombe, auch in guter Hand gehört zu jeder Zeit verdammt. Doch oft schon hat die Welt gewartet und schließlich ist es ausgeartet. Denn sie hat den Kern verkannt. (Quelle: http://www.sueddeutsche.de/kultur/gedicht-zum-konflikt-zwischen-israel-und-iran-was-gesagt-werden-muss-

1.1325809: aufgerufen am 20.12.2013)

Man merkt, hier verwendet der Kommentator bei seiner Confirmation ebenfalls den Kausalitätstopos bzw. den Konsequenztopos und argumentiert ebenfalls in dieselbe Richtung wie der Autor / die erste Fraktion im DaF-Unterricht. Das heißt der Onlinekommentator bzw. die zweite Fraktion im DaF-Unterricht bestätigt in ihrer Confirmation indirekt alle sechs Attribute der Argumente, die in Progymnasmata dafür vorgesehen sind.

Neben dem Kausalitäts- und Konsequenztopos verwendet der Kommentator zudem den »Aus der Vergangenheit-Lehren-ziehen«-Topos bzw. den Analogie-Topos, der mit »doch oft schon ...« eingeleitet wird.

Die dritte Fraktion hat schließlich nun die Aufgabe, die Argumentation des Autors/der ersten und der zweiten Fraktion zu widerlegen (Refutation) und sie in Frage zu stellen. Um sie zu widerlegen muss die dritte Fraktion nun Argumente dafür anführen, dass entweder die Prämissen falsch sind, auf die die Argumente zurückgehen, oder dass die argumentativen Topoi den Schluss von der Prämisse auf die Konklusion nicht erlauben. Das macht der folgende Online-Kommentator mit 
dem Topos des Faktischen, indem er schlicht die Prämisse als falsch und unzutreffend darstellt und daher die ganze Argumentation ad absurdum führt:

»P-MUC 4.4.2012 | 13:32 Uhr

Hab ich was verpasst?

Wann bitte hat Israel je mit einem atomaren Erst-/Vernichtungsschlag gedroht?

Außerdem hat der Iran freiwillig der Kontrolle seines Atomprogramms zugestimmt als es den Atomwaffensperrvertrag unterschrieb, Israel tat das nie. Wobei das zugegebenermaßen wohl sinnvoll wäre ...«

Indem die Prämisse als falsch dargestellt wird, gelingt es dem Online-Kommentator als Stellvertreter der hier vorgeschlagenen dritten Fraktion, die Kausalitätsund Konsequenztopoi zu widerlegen, da sie seiner Meinung nach auf falschen Prämissen beruhen. Im Grundsatz erkennt der Online-Kommentator hier die Stichhaltigkeit dieser Topoi an, jedoch nicht die Richtigkeit der Prämissen. Damit erscheinen die Argumente des Autors als un-verständlich, un-glaubwürdig, unmöglich, un-logisch, un-angemessen und somit un-zweckmäßig. Hier erweitert der Online-Kommentator zudem seine Topoi um den Topos der Vertragstreue und deren Konsequenzen. Demnach sei der Vertragspartner Iran und nicht das vertraglich ungebundene Israel in der Pflicht, sich einer Kontrolle zu unterstellen, während Israel diese Pflicht nicht habe. Natürlich sind andere Widerlegungsmöglichkeiten vorstellbar, die sich ebenfalls in der Kausalität abspielen, wenn man dem Autor/der ersten Fraktion z. B. vorwirft, Ursache und Wirkung zu verwechseln, also dass der Iran Israel bedroht und nicht umgekehrt.

Diese Rollen sollen von den DaF-Lernern unter der Moderation und gegebenenfalls der Korrekturarbeit der DaF-Lehrkraft vorgespielt werden. So kann die Fraktion der Iraner die These vertreten, dass ein atomarer Erstschlag gegen den Iran wahrscheinlich ist, und diese Befürchtung begründen und als Aggression darstellen, oder die weitere These, dass das iranische Volk, anders als die Behauptung des Gedichts, ein mündiges Volk ist und nicht einfach zum organisierten Jubel (vom Ex-Präsidenten Ahmadinedschad) gezwungen wird.

Die Fraktion Israels, die im Gedicht wohl am meisten angesprochen wird, kann ebenfalls ihre eigenen Thesen vertreten, etwa die Gegenbehauptung, dass Israel keine Bedrohung für den Weltfrieden darstellt etc. Bei jeder Fraktion übernehmen die beiden anderen Fraktionen die Rollen der Confirmation und Refutation.

Auf diese Weise können die DaF-Lerner sehr gut aufgezeigt bekommen, wie facettenreich der Einsatz ein und desselben argumentativen Topos sein kann und wie ein argumentierendes Sprechen ein Wettbewerb um die besten argumentativen Topoi sein kann, die jedoch als Ausdruck der Plausibilität und nicht als Ausdruck der Beweiskraft gelten. Topoi sind ihrer Natur nach daher nicht gegen die Widerlegung gefeit. Denn jede Widerlegung lässt sich selbst wiederum auch widerlegen. Wer z. B. behauptet, dass Israel nicht mit einem atomaren Erstschlag gegen Iran gedroht habe, kann schnell mit einem Zitat-Topos widerlegt werden, wenn 
man auf eine Erklärung der israelischen Regierung hinweist, in der sie z. B. tatsächlich direkt oder indirekt mit einem atomaren Erstschlag gegen den Iran droht. Hier werden die DaF-Lerner zudem explizit dafür sensibilisiert, dass für die Produktion eines Arguments auch die Relevanz der gewählten Informationen wichtig ist, da der Confirmation und der Refutation eines Standpunkts unterschiedliche Gegebenheiten zugrunde liegen.

Dass die argumentativen Topoi auch einen Zugang zur zielkulturspezifischen Argumentation sein können, zeigen die kausal verwendeten Geschichts- und Herkunftstopoi in den folgenden zwei Strophen des Gedichts, deren argumentative Analyse, so interessant es auch ist, in diesem engen Rahmen nicht weiterverfolgt werden kann:

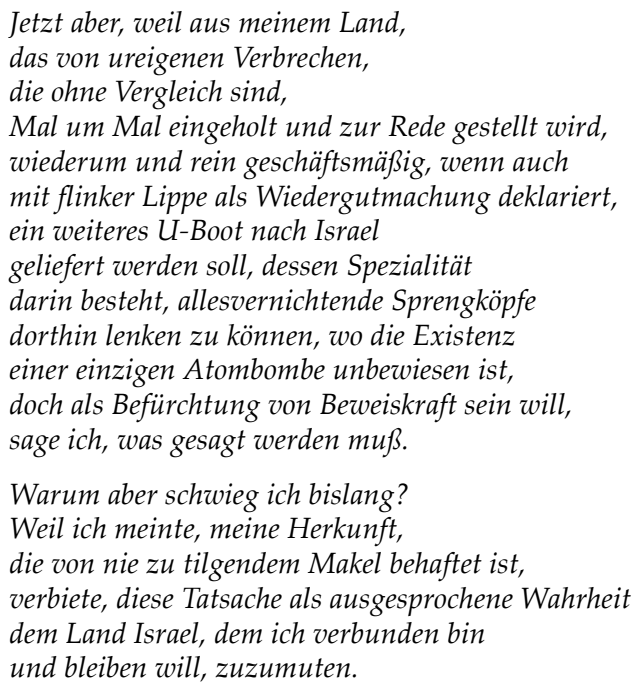

An Wörtern wie »ureigene Verbrechen, die ohne Vergleich sind « und »mit flinker Lippe als Wiedergutmachung deklariert« sendet der Autor - wie bereits oben von Corbett und Connors zur Erweiterung der Effektivität der argumentativen Topoi vorgeschlagen - einen ethischen Appell an seine Kontrahenten und macht klar, dass sein Handeln und seine Argumentation ethisch motiviert sind.

Da es bei dieser ersten Phase der Didaktisierung der argumentativen Topoi nicht auf die Ganzheitlichkeit der argumentierenden Person, auf die Gestik und Mimik und sonstige Körpersprache ankommt, sondern auf die gedankliche Leistung, die sprachliche Formulierung und die Sensibilisierung für das aktuelle Wissen über die (deutsche) Zielkultur und die argumentative Verwendung dieses Wissens, ist es zunächst nicht notwendig, dass man die mündlichen Topoi-Übungen der DaFLerner per Video aufnimmt und diese Videofilme mit dem erforderlichen Zeitauf- 
wand wieder zur Diskussion und Reflexion stellt. Jedoch kann man diese Technik in eine Didaktisierung aufnehmen, wo die Übungen der Rededuelle wirklich von Anfang an spontan konzipiert sind. Dann sind die Redebeiträge wirkliche spontane Rededuelle wie im britischen Parlament und nicht elaborierte Texte zum Vorlesen. Das stellt natürlich an die Sprachkompetenz der DaF-Lerner hohe Anforderungen, die sie möglicherweise erst auf dem Niveau C1 oder C2 erfüllen können.

\section{Die rein schriftliche Vorgehensweise}

Die rein schriftliche Vorgehensweise versteht sich hier nicht als eine "alternative « Option, sondern als »komplementär« und kann daher sowohl vor der mündlichen Option als auch danach realisiert werden. Diese schriftliche Vorgehensweise setzt ebenfalls die oben genannte Vorarbeit am Gedichtstext voraus. Im Fokus des Interesses steht hier nicht die Thematisierung der Schreibkompetenz im DaF-Unterricht im Allgemeinen (vgl. etwa Beaufort 2007; Börner 1989), sondern die Fertigkeit, einen sprachlich und stilistisch korrekten argumentativen Text unter bewusster Berücksichtigung argumentativer Topoi anzufertigen. Auch das Modell des argumentativen Schreibens in der Fremdsprache von Stefan Keller (2012: 93-98) ist nicht gezielt der Analyse und der Produktion von Argumenten und argumentativen Topoi gewidmet, sondern zielt auf den ganzheitlichen Schreibprozess und zeigt auf, dass argumentatives Schreiben in einer Fremdsprache das Resultat vieler Faktoren ist (wie Kompetenzen des Lerners, Weltwissen, Textsortenwissen, Vertrautheit mit rhetorischen Mitteln, Motivation und Überzeugungen, Kreativität und Fleiß sowie Bedingungen der Lernumgebung wie die jeweilige Aufgabenstellung hinsichtlich der Einfachheit und der Komplexität, Vorgaben der jeweiligen Textsorte und der Textorganisation, Adressaten des zu schreibenden Textes, das Lehrkraft-Feedback, das Feedback der Mitlerner sowie die unterstützenden Materialien und Bewertungsmuster). Argumente und Gegenargumente werden in diesem Modell eher beiläufig erwähnt. Dieses Modell ist integrativ orientiert und will den Lehrkräften vermitteln, dass argumentatives Schreiben (und Sprechen) alle weiteren Teilfertigkeiten der DaF-Lerner einschließlich des Transfers aus der Muttersprache bündelt und bündeln sollte, was hier auch mehrfach bestätigt worden ist.

Es sind verschiedene Vorgehensweisen bei der schriftlichen Didaktisierung und der Schulung der DaF-Lerner im Bereich argumentativer Topoi möglich. So kann der Kursleiter zunächst als persönliche Aufgabe seine Klasse damit beauftragen, das Gedicht in einen Artikel umzuwandeln und die hierzu notwendigen sprachlichen Änderungen vorzunehmen, ohne am Inhalt des Gedichts etwas zu ändern. Ein Vortragen von einigen Stichproben aus diesen Artikeln in der Klasse ist angebracht und bietet der Klasse die Chance, sich intensiver mit dem Text zu beschäftigen. 
Der elementare didaktische Schritt aber ist der Einsatz des Instruments des Positionspapiers. Wieder kann die Lehrkraft auf die Idee der Fraktionen des Bundestages zurückgreifen und ihre Klasse in drei Gruppen aufteilen. Jede Gruppe soll ein Positionspapier verfassen und jeweils die Rolle des Autors, der Iraner, der Israelis spielen.

Die schriftliche Aufgabe kann etwa wie folgt lauten:

Stellen Sie sich vor, Sie wären selbst eine der folgenden Parteien:

- der Autor Günter Grass und legen Sie ihre Position dar, warum Sie dagegen sind, dass Deutschland U-Boote an Israel als Wiedergutmachung für das Unrecht an Juden im Dritten Reich liefert;

- ein Vertreter des iranischen Volks und legen Sie Ihre Position dar, ob Sie die vermutete israelische Atommacht als Bedrohung sehen und ob man Israels Atomprogramm wie das iranische Atomprogramm auch kontrollieren soll;

- ein Vertreter Israels und legen Sie dar, warum Israel nicht als Bedrohung des ohnehin brüchigen Weltfriedens angesehen werden kann.

Wenn Sie Ihr Positionspapier zu Ihrer gewählten Rolle geschrieben haben, beantworten Sie bitte folgende vier Fragen:

- Welche argumentativen Topoi haben Sie für die Begründung Ihrer Position benutzt?

- Wie haben Sie den emotionalen und den ethischen Aspekt in Ihre Argumentation integriert?

- Haben Sie ein antizipatives Argument in Ihren Text aufgenommen? Und wenn ja mit welchem Topos?

- Welche Argumente in Ihrem Text sprechen das Publikum der Gegnerpartei an? Sind Ihre argumentativen Topoi publikumsgerecht?

Jede Arbeitsgruppe bzw. jede Fraktion fertigt nun zu einer dieser drei Aufgaben ihr Positionspapier an und beantwortet die vier Fragen dazu ausdrücklich.

- Danach erfolgt das Vortragen jedes Positionspapiers in der Klasse mit einem Kommentar der Lehrkraft zu den Stärken und Schwächen der Argumentation unter der Berücksichtigung der dabei zum Zuge gekommenen Topoi.

- Die zwei anderen Gruppen oder Fraktionen beschreiben, warum der Standpunkt im Positionspapier zu befürworten oder zu widerlegen ist. So bekommt jedes Positionspapier ebenfalls ein dreifaches Feedback durch die eigene Gruppe, durch die beiden anderen Gruppen und durch den Kursleiter.

Durch diese schriftliche Vorgehensweise entsteht auch eine Liste aller im Gedicht und in den mit einbezogenen Online-Kommentaren zum Gedicht verwendeten Topoi. 
Auch wenn bislang davon ausgegangen wurde, dass die Sprachkompetenz im Dienste der Argumentationskompetenz steht, so ist es natürlich auch möglich, dass der Kursleiter auch die Didaktisierung der argumentativen Topoi dazu nützt, die DaF-Lerner für die sprachliche Ausdrucksseite einer erfolgreichen Argumentation zu sensibilisieren.

Die hier vorgestellte Herangehensweise stellt nur einen Vorschlag dar, der im konkreten Fall vielfach modifiziert und erweitert werden kann, auch wenn nicht immer ein hochpolitischer Text erforderlich ist, der viel Hintergrundwissen voraussetzt oder viel Recherchearbeit erforderlich macht.

\section{Fazit}

Es dürfte deutlich geworden sein, dass die Schulung zur argumentativen Kompetenz, insbesondere zur Analyse und zur Verwendung argumentativer Topoi, im DaF-Unterricht weder eine Überfrachtung des Unterrichts noch eine elitäre Gestaltung desselben bedeutet, sondern zu den Kernzielen des DaF-Unterrichts gehört, der die Lernenden auf die friedliche Verständigung mit Angehörigen der deutschen Kulturgemeinschaft vorbereiten soll. Am Beispiel eines literarischen Gedichts, das zugleich als argumentativ gestaltete politische Rede angesehen werden kann, habe ich detailliert darzulegen versucht, wie man das Thema der argumentativen Topoi im DaF-Unterricht bewusst mündlich und schriftlich vom einfachen Einstieg über die Analyse argumentativer authentischer Texte bis zur eigenen Produktion solcher Topoi in eigenen Texten didaktisieren kann und welche Unterrichtsformen dabei als geeignet erscheinen, um den Lernprozess effektiver zu gestalten.

In einem handlungsorientierten kommunikativen DaF-Unterricht ist der Ertrag dieses Ansatzes nicht zu unterschätzen. Die DaF-Lernenden erfahren hier, dass es für jedes Thema typische kontextrelevante Topoi gibt, die nicht nur universal sind, sondern auch zielkultur- und publikumsspezifisch sein sollten, und dass die Produktion erfolgreicher Topoi von der geschickten Auswahl relevanter Informationen aus der Masse verfügbaren Wissens abhängig ist. Durch die Einübung schriftlicher Argumentationsmuster und durch die Reflexion dieser Texte mit den anderen DaF-Lernern sowie mit der Lehrkraft soll den DaF-Lernern vermittelt werden, dass argumentatives Schreiben alle weiteren Fertigkeiten vereint und dass das Auffinden argumentationswirksamer Argumentationsmuster nur einen Bruchteil der Aufgabe der argumentierend schreibenden/sprechenden Person darstellt. Die Freude an der eigenen Produktion argumentativer Texte mit argumentativen Topoi sowie die in der Diskussion mit anderen Lernern und mit der Lehrkraft erlangte Einsicht über den Stellenwert der Sprachbeherrschung des Deutschen in einer Argumentationssituation soll das Interesse an den Sprach- 
strukturen steigern. Wegen des eng absteckten Rahmens war es hier nicht möglich, einen kontrastiven Ansatz zur Didaktisierung von Topoi im DaF-Unterricht zu präsentieren. Es steht aber außer Zweifel, dass im muttersprachlichen Transfer aus der L1 in die L2 ebenfalls ein nicht geringes Potential zur Verbesserung der Didaktisierung der Topoi im DaF-Unterricht steckt.

Es bleibt darauf hinzuweisen, dass die Ausbildung der künftigen DaF-Lehrkräfte im Bereich der Argumentationskompetenz und die bewusste Integration der Didaktisierung der Argumentationsmuster in Lehrwerken z. B. in Texten und Übungen die besten Voraussetzungen sind, damit der moderne kommunikativ orientierte DaF-Unterricht im Zeitalter der globalisierten Kommunikation um diese wichtige Komponente erweitert und ergänzt wird. Gerade die neuen Technologien und das Internet bieten eine Fülle von aktuellen Themen und Diskursen an, die zusätzlich in den Unterricht miteinbezogen werden können und den DaF-Lernern eine Fülle von gängigen thementypischen bzw. zielkulturtypischen Argumentationsmustern anbieten. Auch das E-Learning-Angebot für Sprachen, das Deutsche eingeschlossen, kann für eine sensible Gestaltung von Topoi in argumentativen Texten dahingehend hilfreich sein, dass man mehr universale Topoi integriert und die kulturspezifischen Topoi sensibler gestaltet und didaktisiert. Schließlich wendet sich das Unterrichtsangebot im Internet an globale Nutzer, deren Kultur sehr unterschiedlich ist.

\section{Literatur}

Al-Nasser, Mohammed: Gesprochene Sprache im Deutsch-als-Fremdsprache-Unterricht: Eigenschaften der gesprochenen Sprache in Lehrwerkdialogen. Dissertation Bayreuth, 2011. Zugänglich unter http://d-nb.info/1013138724/34, Abfrage am 30.12.2013.

Aristoteles: Rhetorik. Übersetzt und erläutert von Christof Rapp. Erster und zweiter Halbband. Berlin: Akademie Verlag, 2002.

Beaufort, Anne: College Writing and Beyond: A New Framework for University Writing Instruction. Logan UT: Utah State University Press, 2007.

Börner, Werner: »Didaktik schriftlicher Textproduktion in der Fremdsprache«. In: Antos, Gerd; Krings, Hans P. (Hrsg.): Textproduktion: Ein interdisziplinärer Forschungsüberblick. Tübingen: Niemeyer, 1989, 348-376.

Bornscheuer, Lothar: Topik: Zur Struktur der gesellschaftlichen Einbildungskraft. Frankfurt am Main: Suhrkamp, 1976.

Brinker, Klaus: Linguistische Textanalyse: Eine Einführung in Grundbegriffe und Methoden. Berlin: Erich Schmidt, 1997 (Grundlagen der Germanistik, 29).

Connor, Ulla: »Argumentative Patterns in Student Essays: Cross-cultural Differences«. In: Connor, Ulla; Kaplan, Robert B. (eds.): Writing across Languages: Analysis of L2 text. Reading, MA: Addison-Wesley, 1987, 57-71.

Corbett, Edward P. J.; Connors, Robert J.: Classical Rhetoric for the Modern Student. 4. edition. New York: Oxford University Press, 1999.

Drehe, Iovan: »The Aristotelian Dialectical Topos«, Argumentum 9, 2 (2011), 129-139. 
Drewnowska-Vargáné, Eva: »Topik und rhetorische Stilfiguren im kommunikationskulturellen Vergleich«, Deutsche Sprache 29, 3 (2001), 195-222.

Emmel, Barbara: »Towards a Pedagogy of the Enthymeme: The Roles of Dialogue, Intention, and Function in Shaping Argument «, Rhetoric Review 13, 1 (1994), 132-150.

Europarat: Gemeinsamer Europäischer Referenzrahmen für Sprachen: lernen, lehren, beurteilen. Niveau A1-A2-B1-B2-C1-C2. Berlin: Langenscheidt, 2001.

Flashar, Helmut (Hrsg.): Aristoteles: Rhetorik. Übersetzt und erläutert von Christof Rapp. Erster und zweiter Halbband. Berlin: Akademie Verlag, 2002.

Graff, Richard J:»Topoi/Topics«. In: Fix, Ulla; Gardt, Andreas; Knape, Joachim (Hrsg.): Rhetorik und Stilistik / Rhetoric and Stylistics. 1. Halbband. Berlin: Mouton de Gruyter, 2008, 717-727 (Handbuch der Sprach- und Kommunikationswissenschaft, HSK 31.1).

Heggelund, Kjell T.: »Zur Bedeutung der deutschen Modalpartikeln in Gesprächen unter besonderer Berücksichtigung der Sprechakttheorie und der DaF-Perspektive «, Linguistik online 9, 2/01 Zugänglich unter http://www.linguistik-online.de/9_01/Heggelund.html, Abfrage am 23.12.2013.

Hoffmann, Sarah: Argumentative Strukturen in Sprichwörtern. Bern: Lang, 2012.

Hoppmann, Michael: »Rhetorik des Verstandes (Beweis- und Argumentationslehre)«. In: Fix, Ulla; Gardt, Andreas; Knape, Joachim (Hrsg.): Rhetorik und Stilistik / Rhetoric and Stylistics. 1. Halbband. Berlin: Mouton de Gruyter 2008, 630-645 (Handbuch der Sprachund Kommunikationswissenschaft, HSK 31.1).

Huneke, Hans-Werner; Steinig, Wolfgang: Deutsch als Fremdsprache: Eine Einführung. 3. Auflage. Berlin: Erich Schmidt, 2002.

Keller, Stefan: Integrative Schreibdidaktik: Englisch für die Sekundarstufe. Tübingen: Narr, 2013.

Kennedy, George A. (Übers.): Progymnasmata: Greek Textbooks of Prose Composition and Rhetoric. Leiden: Brill, 2003.

Kienpointner, Manfred: Alltagslogik: Struktur und Funktion von Argumentationsmustern. Stuttgart/Bad Cannstatt: Frommann-Holzboog, 1992, 170-194 (Problemata 126).

Kindt, Walther: 1992: »Argumentation und Konfliktaustragung in Äußerungen über den Golfkrieg «, Zeitschrift für Sprachwissenschaft 11, 2 (1992), 189-215.

Kopperschmidt, Josef: »Pro und Contra im Fernsehen«, Der Deutschunterricht 27, 2 (1975), $42-62$.

Massie, Jessica: »Consideration of Context in the CBI Course Development Process «. In: Jourdenais, Renee-Michele; Springer, Sarah (eds.): Content, Tasks and Projects in the Language Classroom: 2004 Conference Proceedings. Monterey, CA: Monterey Institute of International Studies, 2005, 79-91.

Ockel, Eberhard: »Rede- und Gesprächsfähigkeiten in der gymnasialen Oberstufe: Forderungen der Richtlinien und Umsetzungsvorschläge«. In: Dyck, Joachim u. a. (Hrsg.): Rhetorik: Ein internationales Jahrbuch, Bd. 11. Tübingen: Niemeyer, 1992, 98-114.

Ohlinger, Dalia: Argumentation in der Erst- und Fremdsprache: Pragmalinguistische und grammatikalische Aspekte anhand von Argumentationen deutscher und litauischer Studierender. Frankfurt am Main: Lang, 2003.

Ottmers, Clemens: Rhetorik. Stuttgart: Metzler, 1996 (Sammlung Metzler, 283).

Philipsen, Gerry: Speaking Culturally: Explorations in Social Communication. Albany: State University of New York Press, 1992.

Rapp, Christof: »Topos und Syllogismus in Aristoteles' Topik«. In: Schirren, Thomas; Ueding, Gert (Hrsg.): Topik und Rhetorik. Tübingen: Niemeyer, 2000, 15-35.

Reinert, Johannes: Die Inhaltsangabe im Deutschunterricht: Eine Video- und Interviewstudie zum Umgang mit einer viel diskutierten Textform. Universität Hildesheim, 2012. Zugänglich unter http://nbn-resolving.de/urn:nbn:de:gbv:hil2-opus-1773. Abgefragt am 30.12.2013. 
Rubinelli, Sara: Ars Topica: The Classical Technique of Constructing Arguments from Aristotle to Cicero. Dordrecht: Springer, 2009.

Savignon, Sandra J.: Communicative Competence: Theory and Classroom Practice, Texts and Contexts in Second Language Learning. [Reprint]. Reading, Mass.: Addison-Wesley, 1983.

Schirrmacher, Frank: »Eine Erläuterung: Was Grass uns sagen will«, Frankfurter Allgemeine Zeitung vom 4. April 2012. Zugänglich unter http://www.faz.net/aktuell/feuilleton/debatten/das-israel-gedicht-von-grass/eine-erlaeuterung-was-grass-uns-sagen-will11708120.html. Abfrage am 30.12.2013.

Slomkowski, Paul: Aristotle's Topics. Leiden: Brill, 1997.

Toulmin, Stephen: The Uses of Argument. Cambridge: Cambrige University Press, 1958. Deutsch als: Der Gebrauch von Argumenten. Kronberg/Ts.: Sciptor, 1975.

Ueding, Gerd; Steinbrink, Bernd: Grundriß der Rhetorik: Geschichte, Technik, Methode. 3. Auflage. Stuttgart: Metzler, 1994.

Ueding, Gerd; Steinbrink, Bernd: Grundriss der Rhetorik. Geschichte, Technik, Methode. 5. Auflage. Stuttgart: Metzler, 2011.

Vinçon, Inge: »Argumentationen zwischen Schülern und Lehrer im Deutschunterricht«, Neuere Entwicklungen in Lehre und Lehrerbildung, 1990, 128-137.

Wagner, Tim; Rapp, Christof (Übersetzer): Aristoteles: Topik. Stuttgart: Reclam, 2004.

Wengeler, Martin: »Argumentationstopoi in Diskursen: Zur Nutzung einer rhetorischen Kategorie für die Analyse öffentlichen Sprachgebrauchs«, Der Deutschunterricht 5 (1999), 37-45.

Wengeler, Martin: Topos und Diskurs: Begründung einer argumentationsanalytischen Methode und ihre Anwendung auf den Migrationsdiskurs 1960-1985. Tübingen: Niemeyer, 2003.

Winkler, Iris: Argumentierendes Schreiben im Deutschunterricht: Theorie und Praxis. Frankfurt am Main: Lang, 2003.

Wodak, Ruth et al.: The Discursive Construction of National Identity. Edinburgh: Edinburgh University Press, 1999.

Dr. phil. Abdel-Hafiez Massud

M. A., B. A., Zertifikat DaF/DaZ. Ist Deutsch-Lektor und unabhängiger Postdoc-Researcher. 